\title{
The feasibility of navigation bronchoscopy-guided pulmonary microcoil localization of small pulmonary nodules prior to thoracoscopic surgery
}

\author{
Junxiang Chen ${ }^{1,2,3}$, Xufeng Pan ${ }^{4 \#}$, Chuanjia Gu ${ }^{1,2,3}$, Xiaoxuan Zheng ${ }^{1,2,3}$, Haibin Yuan ${ }^{5}$ Jun Yang ${ }^{4}$, \\ Jiayuan $\operatorname{Sun}^{1,2,3}$
}

${ }^{1}$ Department of Respiratory Endoscopy, Shanghai Chest Hospital, Shanghai Jiao Tong University, Shanghai, China; ${ }^{2}$ Department of Respiratory and Critical Care Medicine, Shanghai Chest Hospital, Shanghai Jiao Tong University, Shanghai, China; ${ }^{3}$ Shanghai Engineering Research Center of Respiratory Endoscopy, Shanghai, China; ${ }^{4}$ Department of Thoracic Surgery, Shanghai Chest Hospital, Shanghai Jiao Tong University, Shanghai, China; ${ }^{5}$ Department of Emergency, Shanghai Chest Hospital, Shanghai Jiao Tong University, Shanghai, China

Contributions: (I) Conception and design: J Sun, J Yang; (II) Administrative support: None; (III) Provision of study materials or patients: J Sun, J Yang; (IV) Collection and assembly of data: J Chen, X Pan, X Zheng, H Yuan, J Yang, J Sun; (V) Data analysis and interpretation: J Chen, X Pan, C Gu, J Yang, J Sun; (VI) Manuscript writing: All authors; (VII) Final approval of manuscript: All authors.

\#These authors contributed equally to this work.

Correspondence to: Jiayuan Sun, MD, PhD. Department of Respiratory Endoscopy, Department of Respiratory and Critical Care Medicine, Shanghai Chest Hospital, Shanghai Jiao Tong University; Shanghai Engineering Research Center of Respiratory Endoscopy, No. 241 West Huaihai Road, Shanghai 200030, China. Email: xkyyjysun@163.com; Jun Yang, MD. Department of Thoracic Surgery, Shanghai Chest Hospital, Shanghai Jiao Tong University, No. 241 West Huaihai Road, Shanghai 200030, China. Email: yj_yangjun@hotmail.com.

Background Accurate preoperative localization of small pulmonary nodules facilitates the rapid and
precise video-assisted thoracoscopic surgery (VATS). This study aims to evaluate the feasibility, safety, and
efficacy of navigation bronchoscopy-guided pulmonary microcoil placement for preoperative pulmonary
nodule localization.

Methods: Twelve lung lesions were simulated by mixing lipiodol in three porcine models. After 1 week, two microcoils per lesion were deployed under bronchoscopic guidance. Computed tomography scans were then performed 1 day, 1 week, 2 weeks, and 4 weeks after the deployment to assess the position of the microcoils relative to the lesions. Surgical resection of the simulated lesions was performed under fluoroscopy 5 weeks after the deployment and the accuracy, stability, and associated complications of the microcoil localization were evaluated. Following this, an exploratory clinical study was conducted on three patients with pure ground-glass pulmonary nodules.

Results: The mean diameter of the twelve simulated lung lesions was $9.55 \pm 2.36 \mathrm{~mm}$, and the mean distance from the pleura to the lesions was $8.29 \pm 2.99 \mathrm{~mm}$. Twenty-four pulmonary microcoils were implanted in the bronchi surrounding the lesions. Four weeks later, the mean distance between the microcoils and the center of the lesions was $16.12 \pm 8.97 \mathrm{~mm}$ and the average migration of the microcoils relative to the baseline position (1 day after implantation) was $3.48 \pm 4.56 \mathrm{~mm}$. All microcoils and target lesions were successfully resected in both the animal experiment and clinical study and no complications, such as pneumothorax, were observed during marker implantation or postoperative follow-up.

Conclusions: The preoperative localization of pulmonary nodules by navigation bronchoscopy-guided microcoil placement is a safe, stable, and effective technique with minimal complication risk. This procedure can assist subsequent thoracoscopic resection.

Keywords: Pulmonary microcoil; pulmonary nodule; navigation bronchoscopy; preoperative localization; videoassisted thoracoscopic surgery (VATS) 
Submitted Oct 19, 2020. Accepted for publication Dec 18, 2020.

doi: $10.21037 /$ tlcr-20-1206

View this article at: http://dx.doi.org/10.21037/tlcr-20-1206

\section{Introduction}

The increasing popularity of low dose chest computed tomography (CT) scan has resulted in a corresponding increased detection of pulmonary nodules (1-3) and video-assisted thoracoscopic surgery (VATS) has become a safe and minimally invasive method for the diagnosis and treatment of small pulmonary nodules (4). However, the localization of lesions during VATS can be difficult, especially for non-visible and non-palpable ground-glass opacities (5). The accurate preoperative localization of small pulmonary nodules facilitates the rapid and precise intraoperative resection of target lesions, which is of great significance for VATS.

Multiple localization techniques, such as fiducial marker placement and dye marking have been proposed (6-10) and each has its own benefits and limitations. The new localization device has been applied to the preoperative localization of pulmonary nodule. Such as 4-hook anchor and scaled suture potentially conducive to reducing the occurrence of displacement or dislodgement (10). Another study was designed a $3 \mathrm{D}$-printed navigational template didn't need CT-guided, the result showed significantly simplifying the localization procedure and decreasing patient radiation exposure (11). At present, the main methods of preoperative positioning of VATS for minimal lung lesion is CT-guided hookwire or microcoil placement, however there are concerns about dislodgement and a high incidence of complications such as pneumothorax and hemorrhage (6). The occurrence of complications is mainly caused by the puncture needle damage the important structures such as pleura, interlobar fissures, pulmonary vessels, and skilled puncture techniques can reduced the incidence of complications. Besides, by offering a less morbid alternative to accurately localize lesions and deploy fiducial markers or indocyanine green within the natural bronchial lumen without damage above anatomical structure, bronchoscopy-guided has reduced procedurerelated complications significantly (8). Bronchoscopic marking is a promising alternative to CT-guided, especially with the emergence of navigation bronchoscopy $(12,13)$.

However, bronchoscopy-guided dye marking may be too large to dispersion or too small to invisible. On the other hand, hookwire and microcoil were initially applied to breast localization and vascular embolization respectively, both of which were not specifically used for pulmonary nodules localization. Therefore, a microcoil was developed specially for the pulmonary, which can anchor around the nodules to assist VATS. In this study, we conducted an animal experiment using porcine models and an exploratory clinical study with small samples to determine the feasibility and safety of implanting pulmonary microcoils under bronchoscopic guidance in the preoperative localization of pulmonary nodules prior to VATS. We present the following article in accordance with the MDAR reporting checklist (available at http://dx.doi.org/10.21037/tlcr-201206).

\section{Methods}

Both animal experiment and clinical study were approved by the Ethics Committee of Shanghai Chest Hospital (No. KS1830 and No. KS1813, respectively). All procedures performed in this study involving human participants were in accordance with the Declaration of Helsinki (as revised in 2013). All patients provided written informed consent and all animals received humane care. Animal experiments were performed in compliance with china national or institutional guidelines for the care and use of animals. A flow chart of the animal experiment is shown in Figure 1. All procedures were performed under general intravenous anesthesia and endotracheal intubation, as described in Yuan et al. [2019] (14).

\section{Animal experiment}

\section{Simulation of the lung lesions}

In this component of the study, three male white pigs (Shanghai JiaGan Biotechnology Co. Ltd., Shanghai, China) weighing approximately $55 \mathrm{~kg}$ each were subject to 1 week of adaptive feeding. A chest CT scan (Philips Brilliance 64 multi-slice spiral CT, Philips Healthcare, Amsterdam, the Netherlands) was then obtained to exclude those with pulmonary inflammation. Following this, predetermined sites in the right lower lung (RLL), right upper lung (RUL), left lower lung (LLL), and left upper lung (LUL) were 


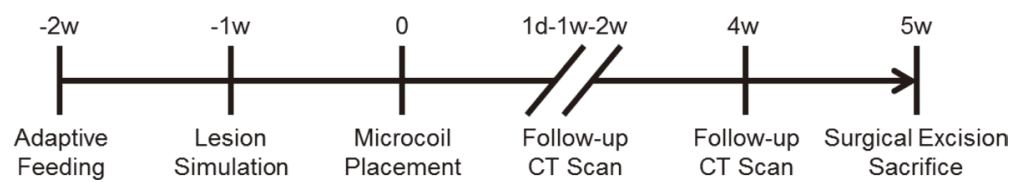

Figure 1 Flow chart of the animal experiment. Simulation of lung lesions in porcine models took place after 1 week of adaptive feeding. One week later, the localization of simulated lung lesions with pulmonary microcoils took place and chest CT scans was repeated 1 day, 1 week, 2 weeks, and 4 weeks after the placement of microcoils. Both lung lesions and pulmonary microcoils were surgically resected 5 weeks after the localization. CT, computed tomography.

percutaneously punctured by an experienced interventional physician using a 22-Gauge Chiba biopsy needle (Argon Medical Devices, Inc., Illinois, USA). A 1:1 mixture of lipiodol (Iodinated Oil Injection, Luyin, Yantai, China) and biogel (Medical Adhesive, Baiyun, Guangzhou, China) was then injected. The chest CT scan was repeated 1 week after injection to confirm the shape and location of the simulated lung lesions.

\section{Pulmonary microcoil placement and follow-up}

The simulated lesions were first localized using virtual bronchoscopic navigation (VBN; DirectPath, Olympus, Tokyo, Japan), which allowed a planned route to the target lesion to be established. The delivery sheath was then placed at the bronchus surrounding the lesion using the bronchoscopic working channel (BF-P290, Olympus, Tokyo, Japan) and intraoperative fluoroscopy (Philips Veradius Unity, Philips Healthcare, Amsterdam, Netherlands) was performed under virtual guidance to confirm that the delivery sheath was in the vicinity of the target lesion. Two pulmonary microcoils (Lunghealth Medtech Co. Ltd., Changzhou, China) per lesion were then deployed under fluoroscopy. Regular CT scans were performed 1 day, 1 week, 2 weeks, and 4 weeks after the procedure to observe for complications and measure the relative distance between the microcoils and the center of the lesion.

\section{Surgical resection}

Surgical resection of the simulated lesion was performed 5 weeks after pulmonary microcoil placement. The CT images were reconstructed as a maximum intensity projection (MIP) image of the microcoils and lesions on the same plane to assess the relative position prior to resection. During the resection, fluoroscopy was used to visualize and guide the surgical resection and excision of each complete microcoil and lesion. The excised specimen was then dissected to confirm that the entire microcoil and lesion had been removed and the results were obtained to determine the success rate of the localization and resection.

\section{Clinical study}

In his component of the study, we enrolled three patients with pure ground-glass pulmonary nodules who were considered as candidates for VATS sub-lobectomy. The patients first underwent general anesthesia and their nodules localized using electromagnetic navigation bronchoscopy (ENB; LungCare navigation system, LungCare Medical Technologies Ltd., Inc., Suzhou, China). After navigation, the locatable electromagnetic guide was removed, and the extended working channel sheath left in place. Following this, three-dimensional reconstruction using cone-beam CT (Discovery IGS 730, GE Healthcare, Boston, USA) was performed to confirm that the sheath had again reached the vicinity of the lesion, after which the microcoil was deployed alongside the nodule under fluoroscopy.

After microcoil placement, the single-lumen tube was replaced with a double-lumen tube, the patient placed in the lateral position on the healthy side, and one lung ventilated. After confirming the location of the lesion by visualizing the microcoils with fluoroscopy, wedge resection or segmentectomy under VATS was performed. Excision of the complete microcoil and nodule was confirmed fluoroscopically. Intraoperative frozen section examination was then performed, and resected specimens were sent to the pathology department for further analysis.

\section{Statistical analysis}

Data were summarized by descriptive statistics, including mean, standard deviation, median, minimum, and maximum values for continuous variables. All analyses were performed using Microsoft Excel (Microsoft, Seattle, USA). 


\section{Results}

\section{Animal experiment}

Twelve simulated lung lesions were constructed by percutaneous puncture using a mixture of lipiodol and biogel. These were evenly distributed in the RLL, RUL, LLL, and LUL of the three pigs. The simulation process is shown in Figure 2. The mean diameter of the lesions was $9.55 \pm 2.36 \mathrm{~mm}$, and the mean distance from the pleura was $8.29 \pm 2.99 \mathrm{~mm}$. During the percutaneous puncture, a mild pneumothorax in the lower lung (data not displayed) occurred in three lesions $(25 \%, 3 / 12)$, but tube drainage was not required. There were no other complications including hemorrhage and chest CT at re-examination 1 week later showed full pneumothorax absorption.

Two kinds (one-tailed and double-tailed) of pulmonary microcoils were respectively used in the animal experiment and clinical trial (Figure $3 A$ ). In the porcine models, two microcoils with one-tailed gold seed attachment (top left in Figure $3 A$ ) were deployed in each simulated lesion under the guidance of $\mathrm{VBN}$ and fluoroscopy (Figure $3 B, C, D, E, F$ ). In total, 24 pulmonary microcoils were implanted in the bronchi surrounding the simulated lesions. The chest CT was repeated 1 day, 1 week, 2 weeks, and 4 weeks after deployment, and the distance between the microcoils and the center of the lesion was again measured, as shown in Figure 4. The average distance between the microcoils and the center of the lesions was $16.12 \pm 8.97 \mathrm{~mm} 4$ weeks after the deployment, and the average dislodgement was $3.48 \pm 4.56 \mathrm{~mm}$. The overall migration 4 weeks after the implantation is summarized as follows: 21 microcoils $(87.5 \%)$ with a migration of $0-5 \mathrm{~mm} ; 2(8.33 \%)$ with a migration of $5-10 \mathrm{~mm} ; 1(4.17 \%)$ with a migration $>10 \mathrm{~mm}$. Complications, including pneumothorax and hemorrhage were not observed during microcoil placement and postprocedure follow-up.

All 12 simulated lung lesions were successfully resected, with a surgical success rate of $100 \%$. The success rate of preoperative localization with pulmonary microcoils was also $100 \%$. Representative images are shown in Figure 5.

\section{Clinical study}

Three female patients with an average age of 55 years participated in the clinical study. All patients had pure ground-glass opacity pulmonary nodules listed for sublobectomy; two with lesions in the RUL and one with a lesion in the LLL. The mean maximum diameter of the nodules was $12.58 \mathrm{~mm}$ and the mean distance to the pleura was $10.55 \mathrm{~mm}$. Of the three, one had a bronchus leading to it, one had a bronchus approaching it, and one did not have a bronchus passing through it. The guided sheath was placed at the target position under the guidance of ENB. After confirmation by cone-beam CT reconstruction, the pulmonary microcoil was deployed around the nodule. A second cone-beam CT scan was obtained to confirm microcoil placement and no complications such as pneumothorax were observed. VATS was performed simultaneously, and the results revealed the effective and successful preoperative localization of the nodules. Wedge resection was performed in two cases, and segmental resection in the other, with pathology revealing two cases of adenocarcinoma-in-situ, and one of microinvasive adenocarcinoma. The representative cases are shown in Figure 6. No increased thoracic drainage liquid and abnormal color, fever, atelectasis or other conditions were found after the operation, and the patient has not complained of discomfort since surgery 1 month.

\section{Discussion}

Intraoperative identification and resection of suspected malignant pulmonary nodules, especially non-visible and non-palpable ground-glass opacities, is a challenging issue during VATS (5). Precise preoperative localization is the key to improving the success rate and safety of thoracoscopic sub-lobectomy resection. Transcutaneous and transbronchial marking are the two most used pathways for pulmonary nodules localization $(6-8,15,16)$. Since the former method is associated with a relatively high incidence of complications such as pneumothorax and hemorrhage, the latter may be a promising alternative $(6,17-19)$. In this study, we investigated the feasibility, stability, and efficacy of navigation bronchoscopy-guided pulmonary microcoil localization of small pulmonary nodules prior to VATS.

Fiducial markers were initially used for tracking lesions in gated stereotactic body radiotherapy (20-23) but more recently they have also been used for localizing lung tumors to aid VATS $(7,15,24)$. Of the two types of markers, studies have revealed that the linear type have a relatively high dislodgement rate compared with the coiled type (21). In the present study, microcoils were used because they expand after deployment and wedge themselves by recoil forces into the surrounding bronchial wall.

Microcoils are also manufactured with different diameters which allow them to be placed into different sizes 
Preoperative
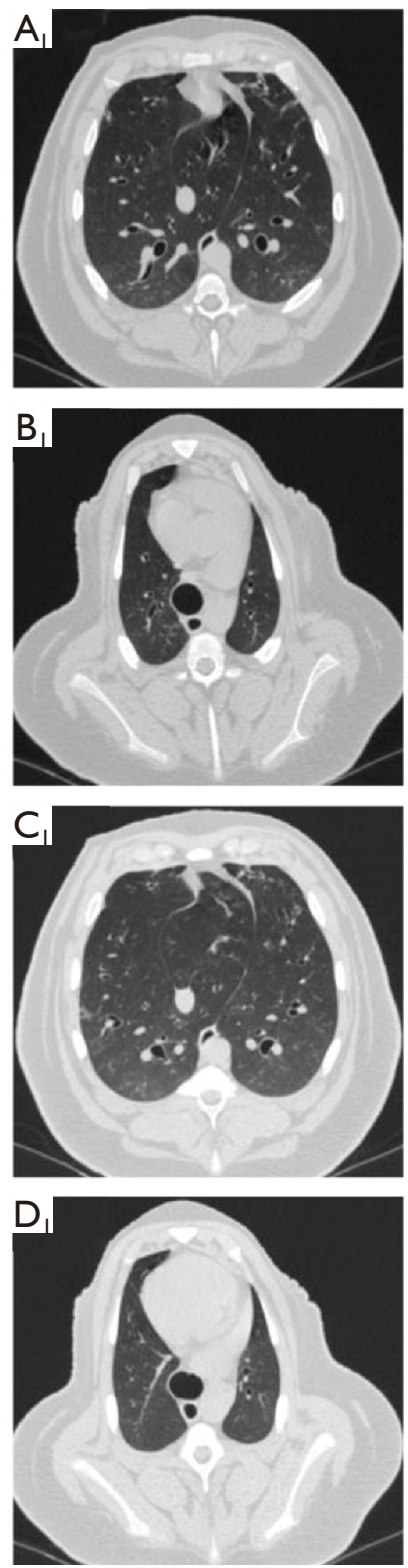

Intraoperative
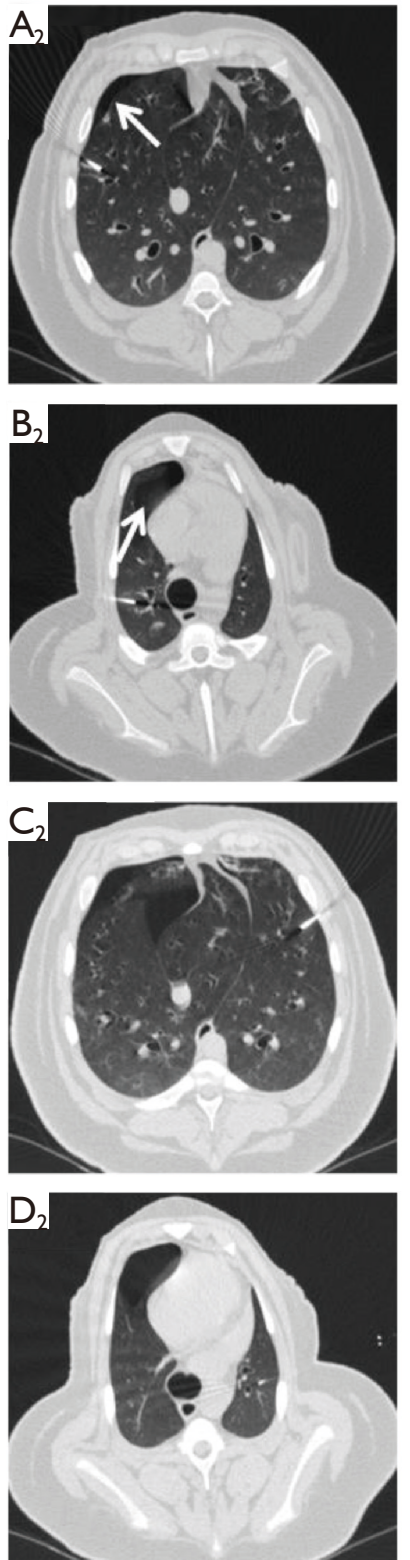

Postoperative
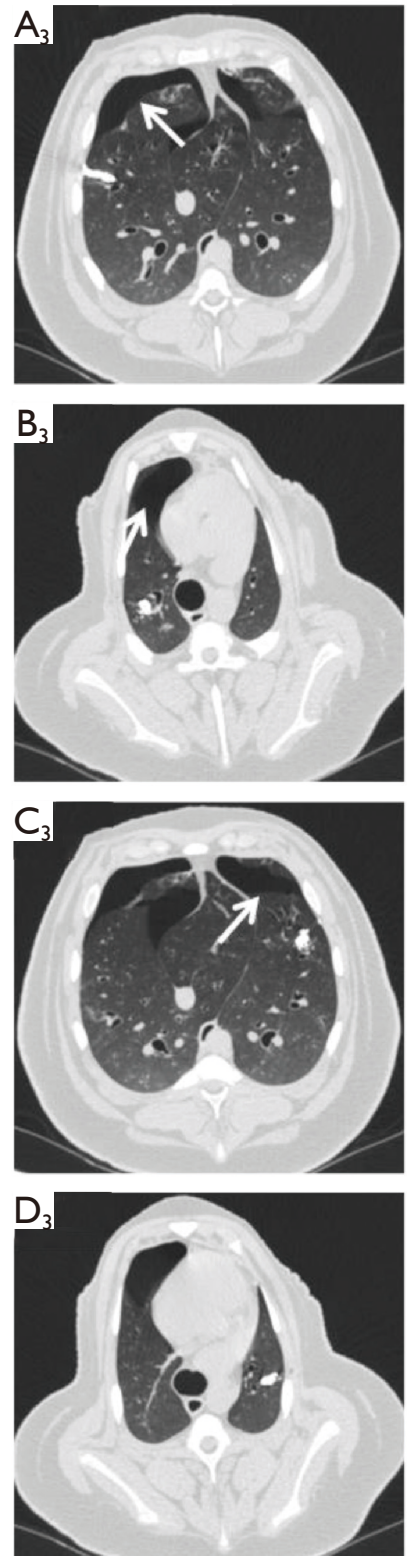

After $1 \mathrm{w}$
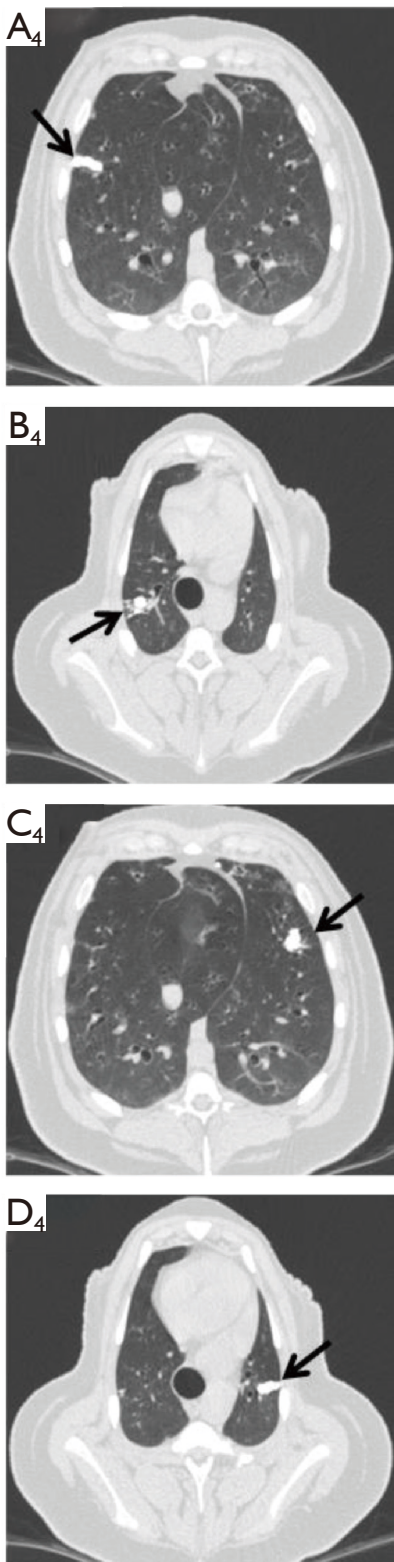

Figure 2 Simulation process of lung lesions in porcine models through percutaneous injection of mixed lipiodol into different lobes. (A1,B1,C1,D1) CT scan of different lobes before puncture. (A2,B2,C2,D2) Needle reaching the predetermined target position in different lobes. (A3,B3,C3,D3) CT scan immediately after the injection of mixed lipiodol. (A4,B4,C4,D4) CT scan of simulated lung lesions 1 week after injection. White arrows represent the pneumothorax caused by the puncture, and black arrows represent the lung lesions simulated by the injection of mixed lipiodol. CT, computed tomography; LLL, left lower lung; LUL, left upper lung; RLL, right lower lung; RUL, right upper lung.

of bronchial lumen (25). To our knowledge, this is the first systematic study of pulmonary microcoil localization in an animal experiment and clinical study under bronchoscopic guidance. The feasibility of pulmonary nodule localization using microcoils with one-tailed gold seed attachment under bronchoscopy was first tested in the porcine model. 

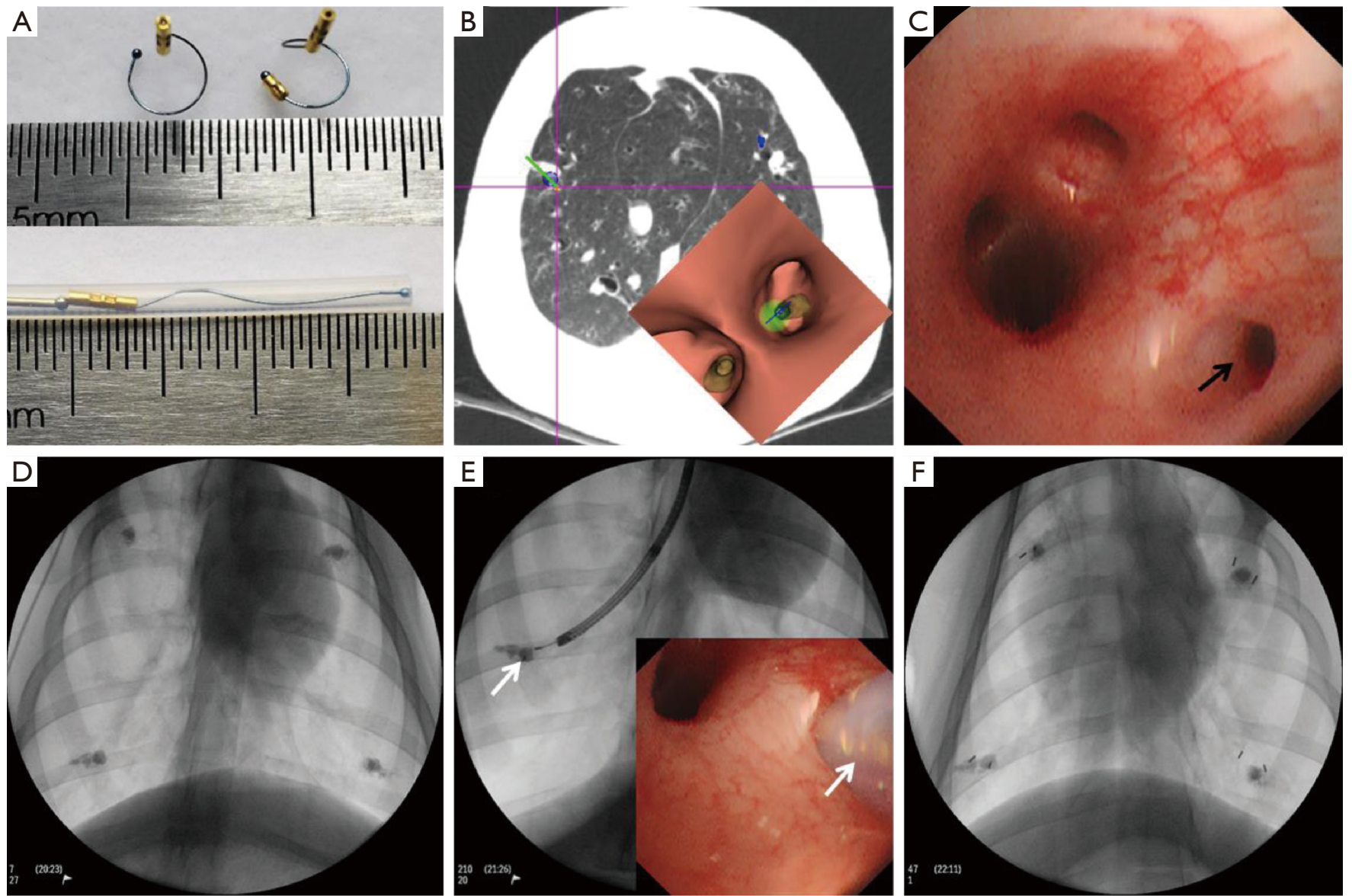

Figure 3 Pulmonary microcoil placement for simulated lesion localization under the guidance of VBN and fluoroscopy in an in-vivo porcine model. (A) Deployed coiled pulmonary microcoils (top) with one-tailed gold seed attachment (left) and double-tailed gold seed attachment (right) to a coiled nitinol wire. Flattened coil pulmonary microcoil loaded in tip of delivery sheath (bottom). (B) VBN provided guidance to the bronchi surrounding the lesion. (C) The target bronchus (black arrow) under white light bronchoscopy. (D) Fluoroscopy of simulated lung lesions before microcoil placement. (E) Placement of pulmonary microcoil (white arrows) under the guidance of fluoroscopy. (F) Fluoroscopy immediately after placement showing the pulmonary microcoils are all located around the simulated lung lesions. VBN, virtual bronchoscopic navigation.

Based on these results, a new type of microcoil with doubletailed gold seed attachment was employed in the clinical study (Figure 6), as this was more conducive to observing the deployment procedure.

Previous study has demonstrated that ENB is a wellestablished technique with excellent accuracy and safety (26). In this study we used VBN as a navigation mode in porcine solid nodules (fluoroscopy-detectable) and ENB in human pure ground-glass nodules (fluoroscopy-undetectable) and the results show that both methods could localize pulmonary nodules successfully. Whilst there was no difference in localization success rate between VBN and ENB in our study, the potential advantage of ENB may be that it gives real time display of the distance from the fiducial marker to the lesion, facilitating determination of the surgical resection boundary, although this needs to be further explored (27). Our results show that pulmonary nodule localization and resection can be conducted simultaneously. This reduces anesthesia time and is more convenient for patients when compared to conducting multiple procedures at different times (24). In addition, the integration of ENB with cone-beam CT ensured the accuracy of localization through immediate feedback in clinical studies. These adjunctive techniques help determine the appropriate 
RLL

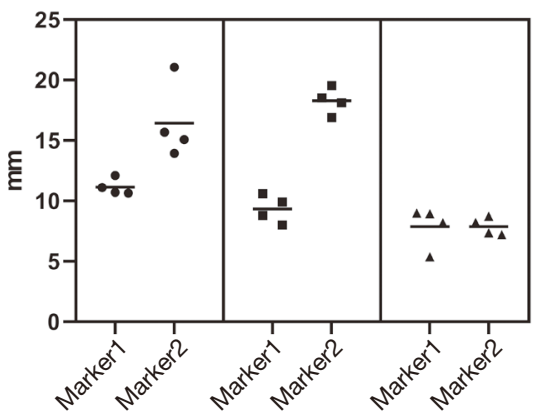

LLL

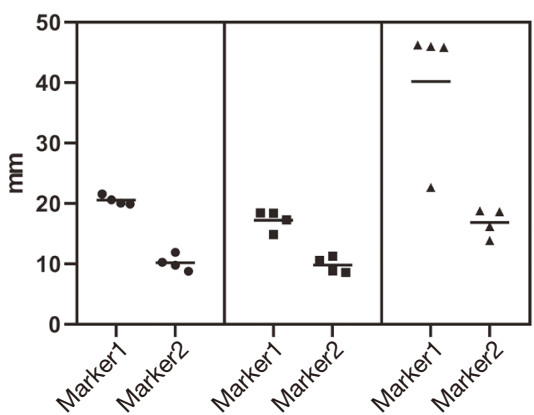

RUL

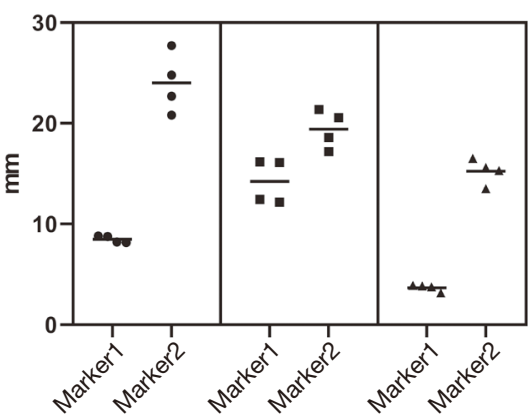

LUL

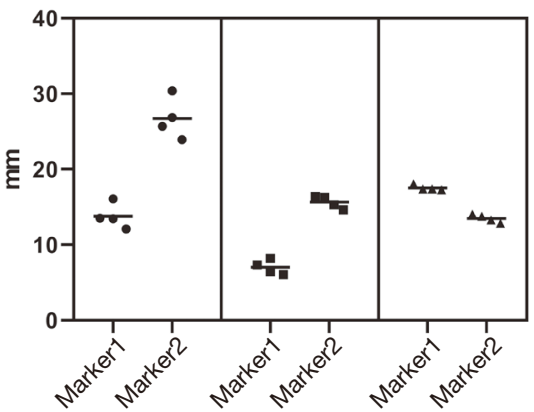

Figure 4 Distance from the simulated lung lesions to pulmonary microcoils at different time points (1 day, 1 week, 2 weeks, and 4 weeks) after microcoil placement. Twelve lung lesions were simulated in three pigs, and two pulmonary microcoils were placed surrounding each lesion. Chest CT scan was performed 1 day, 1 week, 2 weeks, and 4 weeks after the placement to measure the distance between microcoils and lesions. CT, computed tomography; LLL, left lower lung; LUL, left upper lung; RLL, right lower lung; RUL, right upper lung.

placement of microcoils and correct discrepancies between preoperative CT scan and bronchoscopic images (24).

This study has revealed several important factors in the placement of pulmonary microcoils. Firstly, placement can be made in any lobe, which was demonstrated in both the animal experiment and clinical components. Secondly, placement is not restricted by an accessible bronchus to the lesion because it can be deployed within or around the lesion, distinguishing this from transbronchial lung biopsy, which relies on an accessible bronchus to the lesion (12). Thirdly, a ratio of one pulmonary microcoil per lesion was sufficient to achieve precise preoperative localization although multiple microcoils should be used when more accurate localization or stereoscopic coordinates are needed (21). Finally, in order to ensure the precise positioning of the pulmonary nodules, the thin slice CT images should be carefully checked, and the bronchial path leading to or adjacent to the lesion should be optimized before localization. The pulmonary microcoil should be placed around the lesion as far as possible, and the distance from the lesion should be
$<2 \mathrm{~cm}$ to ensure that there is enough excision edge and no microcoil remains. During surgery, X-ray would be done for collapsed lung to confirm the position relationship between pulmonary microcoil and ribs. Next, searching the microcoil under fluoroscopy or touching with fingers, then the lung tissue containing the microcoil should be clipped with oval forceps, and confirmed by X-ray (see Figure $6 E$ ). The lesion was then complete resected.

Our study also revealed that the microcoils yielded high stability and safety for preoperative localization. The average distance between the microcoils and the center of the lesions was $16.12 \pm 8.97 \mathrm{~mm} 4$ weeks after implantation. This shows an average migration of $3.48 \pm 4.56 \mathrm{~mm}$ from the day of implantation. Imaging follow up at one month showed a $100 \%$ retention rate, confirming durable stability, and verifying the results of previous studies. This indicates that resection can take place long after microcoil implantation. All microcoils and lesions were successfully removed, and no dislodgement was observed during the surgery. Most importantly, microcoils placed via ENB had 
MIP Model
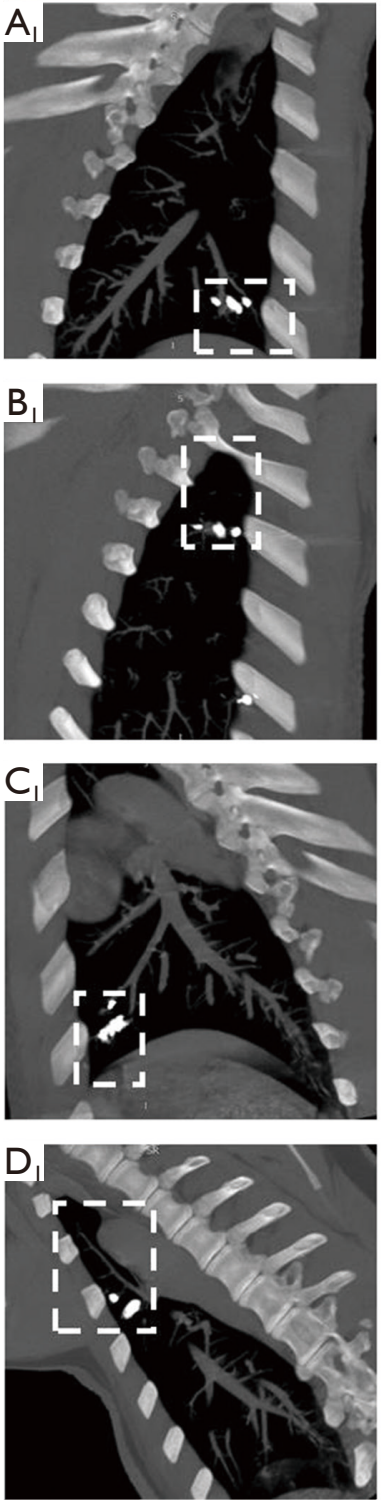

Hemostat
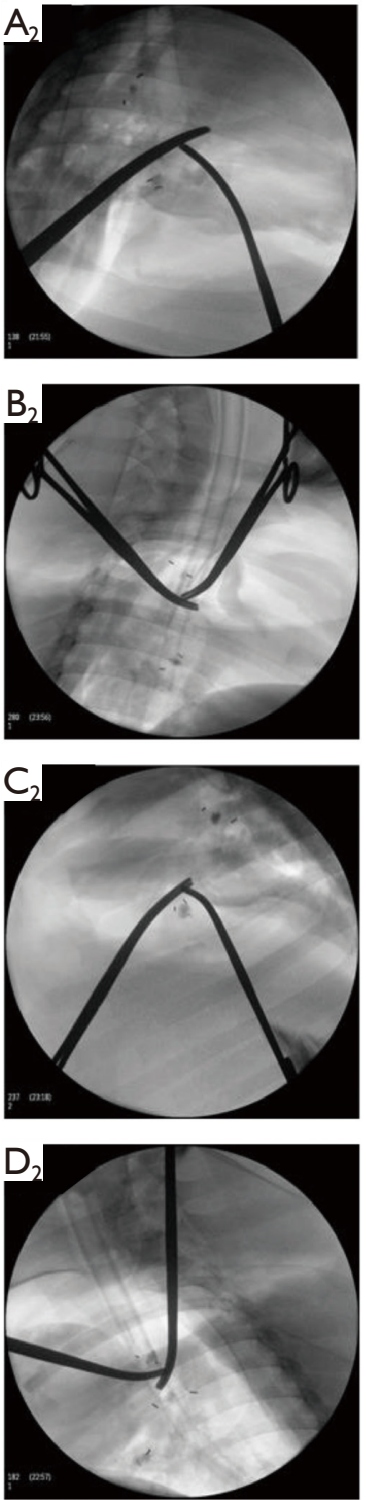

Postoperative
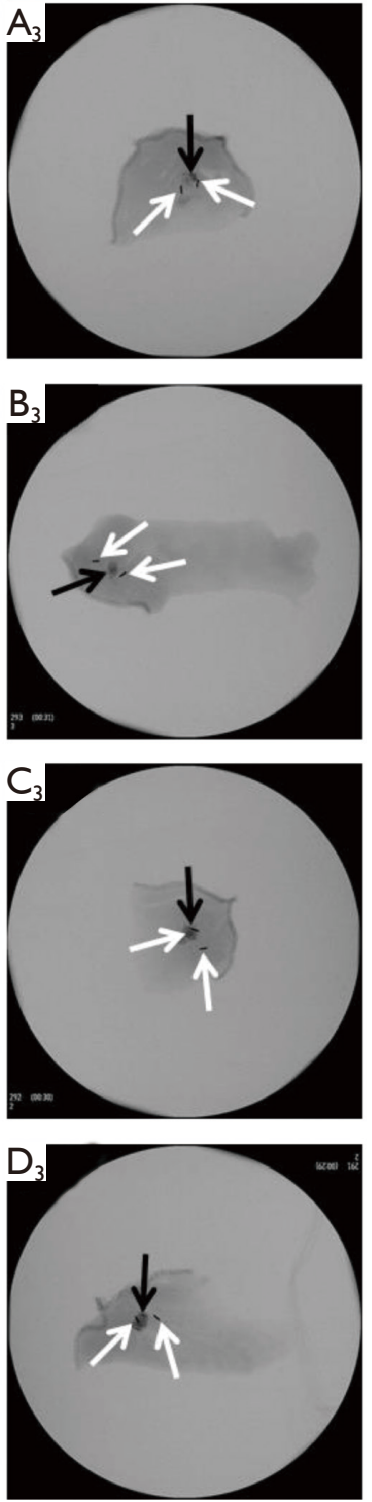

Dissection
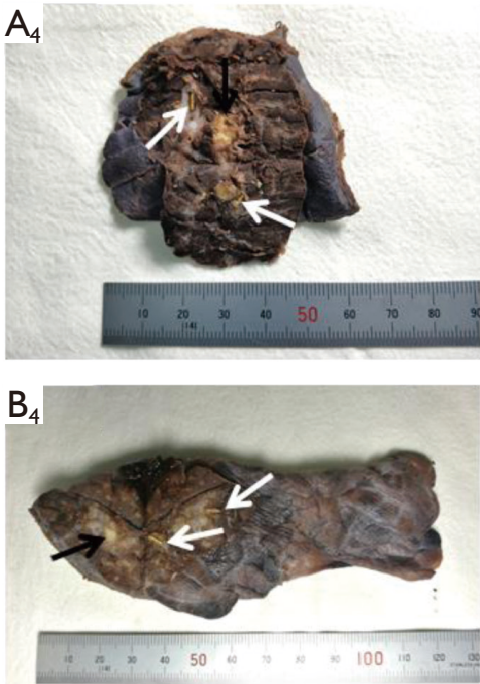

$\mathrm{C}_{4}$

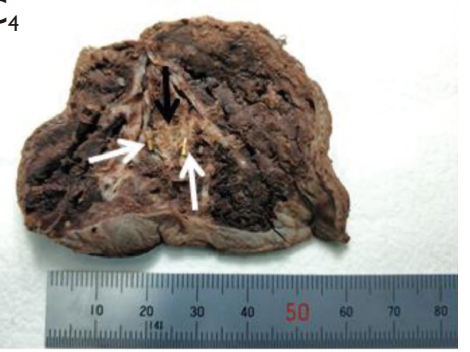

$\mathrm{D}_{4}$

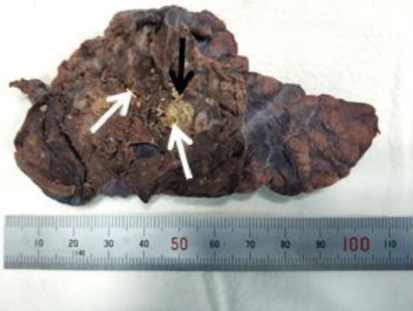

Figure 5 Surgical resection of simulated lung lesions. (A1,B1,C1,D1) Reconstructed preoperative MIP images of pulmonary microcoils and simulated lung lesions on the same plane. (A2,B2,C2,D2) Fluoroscopy confirming both lesions and microcoils are located within the lung tissue to be resected. (A3,B3,C3,D3) Fluoroscopy of wedge resection specimen confirming all microcoils (white arrows) and simulated lesions (black arrows) have been removed. (A4,B4,C4,D4) Surgical specimen containing both lesions (black arrows) and the microcoils (white arrows). MIP, maximum intensity projection; LLL, left lower lung; LUL, left upper lung; RLL, right lower lung; RUL, right upper lung.

a significantly lower incidence rate of pneumothorax as compared with those placed percutaneously (28). No other procedure-related complications occurred in our study, which is consistent with others $(12,13,20,23)$.

But this study was limited by its small sample size and absence of a control group. Larger, multicenter, prospective, randomized controlled clinical trials are needed to further evaluate the advantages of the pulmonary microcoils to other devices. Another limitation of the technique was the surgeon and the patient need to be exposed to small doses of radiation during image-guided VATS.

In conclusion, the preliminary results of this animal 

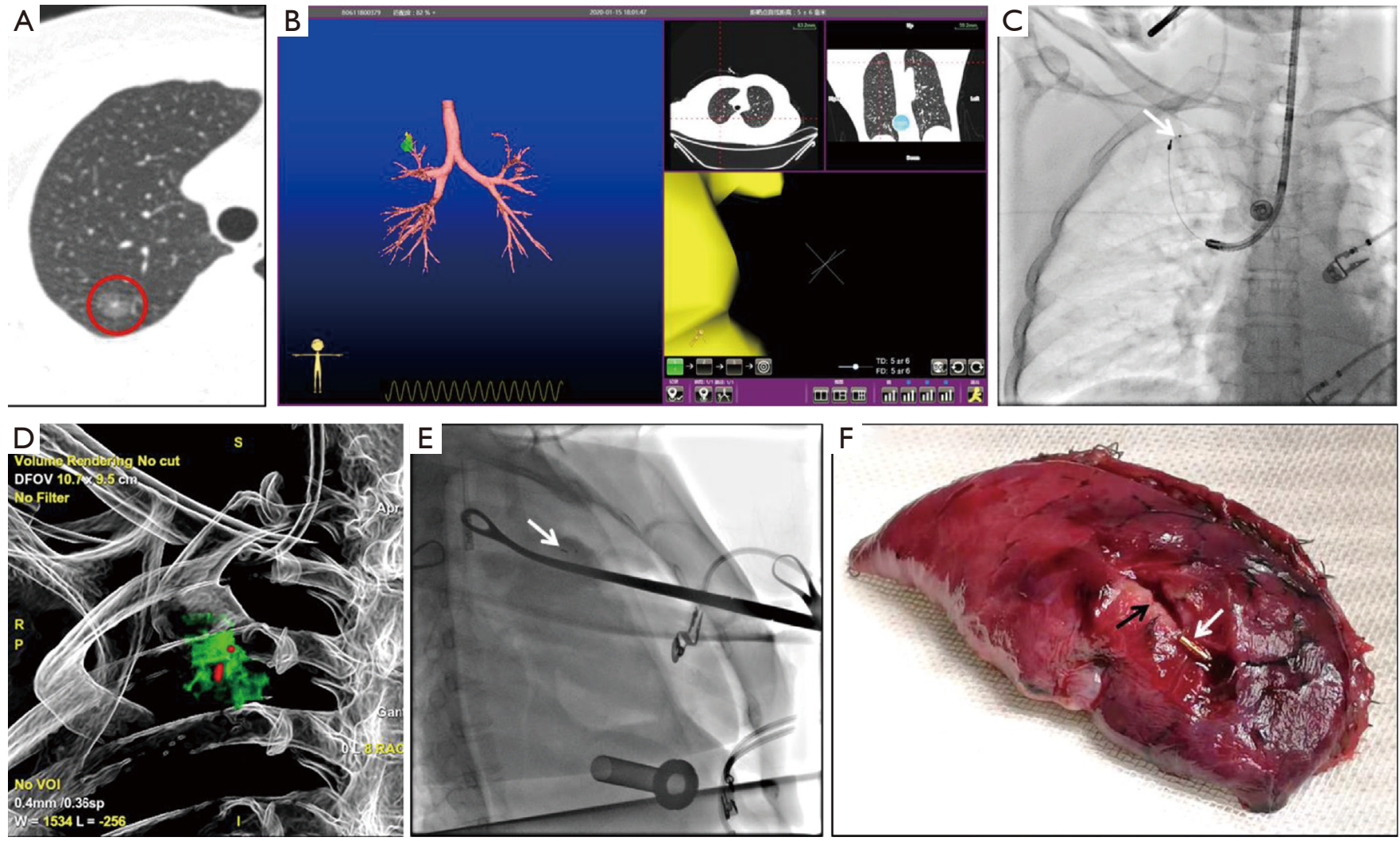

Figure 6 Pulmonary microcoils placed under ENB and cone-beam CT guidance and subsequent VATS resection. (A) CT scan showing a pure ground-glass pulmonary nodule located in the upper lobe of the right lung. (B) ENB showing the accurate access to the target pulmonary nodule. (C) Pulmonary microcoil (white arrow) placed under the intraoperative fluoroscopy. (D) Three-dimensional reconstruction of the target pulmonary nodule and pulmonary microcoil using cone-beam CT scan. (E) Fluoroscopy confirming the microcoil (white arrow) is included in the lung parenchyma to be resected. (F) Surgical specimen containing both tumor (black arrow) and pulmonary microcoil (white arrow). CT, computed tomography; VATS, video-assisted thoracoscopic surgery; ENB, electromagnetic navigation bronchoscopy.

experiment and exploratory clinical study show that navigation bronchoscopy-guided placement of pulmonary microcoils before VATS is a feasible, safe and effective strategy for the preoperative localization of pulmonary nodules. This may have considerable clinical potential in the future treatment of lung cancer.

\section{Acknowledgments}

Funding: The study was supported by the National Key R\&D Program of China (2017YFC0112700), Shanghai Municipal Education Commission-Gaofeng Clinical Medicine Grant Support (20181815), Shanghai Municipal Health and Medical Talents Training Program (2018BR09), and Clinical Research Plan of SHDC (16CR3007A).

\section{Footnote}

Reporting Checklist: The authors have completed the MDAR reporting checklist. Available at http://dx.doi.org/10.21037/ tlcr-20-1206

Data Sharing Statement: Available at http://dx.doi. org/10.21037/tlcr-20-1206

Conflicts of Interest: All authors have completed the ICMJE uniform disclosure form (available at http://dx.doi. org/10.21037/tlcr-20-1206). The authors have no conflicts of interest to declare.

Ethical Statement: The authors are accountable for all 
aspects of the work in ensuring that questions related to the accuracy or integrity of any part of the work are appropriately investigated and resolved. Both animal experiment and clinical study were approved by the Ethics Committee of Shanghai Chest Hospital (No. KS1830 and No. KS1813, respectively). All procedures performed in this study involving human participants were in accordance with the Declaration of Helsinki (as revised in 2013). All patients provided written informed consent and all animals received humane care. Animal experiments were performed in compliance with china national or institutional guidelines for the care and use of animals.

Open Access Statement: This is an Open Access article distributed in accordance with the Creative Commons Attribution-NonCommercial-NoDerivs 4.0 International License (CC BY-NC-ND 4.0), which permits the noncommercial replication and distribution of the article with the strict proviso that no changes or edits are made and the original work is properly cited (including links to both the formal publication through the relevant DOI and the license). See: https://creativecommons.org/licenses/by-nc-nd/4.0/.

\section{References}

1. Church TR, Black WC, Aberle DR, et al. Results of initial low-dose computed tomographic screening for lung cancer. N Engl J Med 2013;368:1980-91.

2. de Koning HJ, van der Aalst CM, de Jong PA, et al. Reduced lung-cancer mortality with volume CT screening in a randomized trial. N Engl J Med 2020;382:503-13.

3. Vlahos I, Stefanidis K, Sheard S, et al. Lung cancer screening: nodule identification and characterization. Transl Lung Cancer Res 2018;7:288-303.

4. Sihoe AD. Video-assisted thoracoscopic surgery as the gold standard for lung cancer surgery. Respirology 2020;25 Suppl 2:49-60.

5. McDermott S, Fintelmann FJ, Bierhals AJ, et al. Imageguided preoperative localization of pulmonary nodules for video-assisted and robotically assisted surgery. Radiographics 2019;39:1264-79.

6. Park CH, Han K, Hur J, et al. Comparative effectiveness and safety of preoperative lung localization for pulmonary nodules: a systematic review and meta-analysis. Chest 2017;151:316-28.

7. Abbas A, Kadakia S, Ambur V, et al. Intraoperative electromagnetic navigational bronchoscopic localization of small, deep, or subsolid pulmonary nodules. J Thorac
Cardiovasc Surg 2017;153:1581-90.

8. Kuo SW, Tseng YF, Dai KY, et al. Electromagnetic navigation bronchoscopy localization versus percutaneous CT-guided localization for lung resection via videoassisted thoracoscopic surgery: a propensity-matched study. J Clin Med 2019;8:379.

9. Park CH, Lee SM, Lee JW, et al. Hook-wire localization versus lipiodol localization for patients with pulmonary lesions having ground-glass opacity. J Thorac Cardiovasc Surg 2020;159:1571-1579.e2.

10. Fan L, Yang H, Yu L, et al. Multicenter, prospective, observational study of a novel technique for preoperative pulmonary nodule localization. J Thorac Cardiovasc Surg 2020;160:532-539.e2.

11. Zhang L, Wang L, Kadeer X, et al. Accuracy of a 3-Dimensionally Printed Navigational Template for Localizing Small Pulmonary Nodules: A Noninferiority Randomized Clinical Trial. JAMA Surg 2019;154:295-303.

12. Bowling MR, Folch EE, Khandhar SJ, et al. Fiducial marker placement with electromagnetic navigation bronchoscopy: a subgroup analysis of the prospective, multicenter NAVIGATE study. Ther Adv Respir Dis 2019;13:1753466619841234.

13. Belanger AR, Burks AC, Chambers DM, et al. Peripheral lung nodule diagnosis and fiducial marker placement using a novel tip-tracked electromagnetic navigation bronchoscopy system. J Bronchology Interv Pulmonol 2019;26:41-8.

14. Yuan HB, Wang XY, Sun JY, et al. Flexible bronchoscopyguided microwave ablation in peripheral porcine lung: a new minimally-invasive ablation. Transl Lung Cancer Res 2019;8:787-96.

15. Andrade RS. Electromagnetic navigation bronchoscopyguided thoracoscopic wedge resection of small pulmonary nodules. Semin Thorac Cardiovasc Surg 2010;22:262-5.

16. Xie F, Zhang J, Cao L, et al. Design of a prospective, multicenter, and cohort study of an innovative electromagnetic navigation bronchoscopy in diagnosing pulmonary nodules among Chinese population. J Thorac Dis 2019;11:5592-600.

17. Obeso A, Ng CSH. Electromagnetic navigation bronchoscopy in the thoracic hybrid operating room: a powerful tool for a new era. J Thorac Dis 2018;10:S764-8.

18. Hsu PK, Chuang LC, Wu YC. Electromagnetic navigation-guided preoperative localization of small malignant pulmonary tumors. Ann Thorac Surg 2020;109:1566-73.

19. Hung CT, Chen CK, Chang YY, et al. Electromagnetic 
navigation-guided versus computed tomographyguided percutaneous localization of small lung nodules before uniportal video-assisted thoracoscopic surgery: a propensity score-matched analysis. Eur J Cardiothorac Surg 2020;58:i85-i91.

20. Anantham D, Feller-Kopman D, Shanmugham LN, et al. Electromagnetic navigation bronchoscopy-guided fiducial placement for robotic stereotactic radiosurgery of lung tumors: a feasibility study. Chest 2007;132:930-5.

21. Schroeder C, Hejal R, Linden PA. Coil spring fiducial markers placed safely using navigation bronchoscopy in inoperable patients allows accurate delivery of CyberKnife stereotactic radiosurgery. J Thorac Cardiovasc Surg 2010;140:1137-42.

22. Nabavizadeh N, Zhang J, Elliott DA, et al. Electromagnetic navigational bronchoscopy-guided fiducial markers for lung stereotactic body radiation therapy: analysis of safety, feasibility, and interfraction stability. J Bronchology Interv Pulmonol 2014;21:123.

23. Minnich DJ, Bryant AS, Wei B, et al. Retention rate of electromagnetic navigation bronchoscopic placed fiducial markers for lung radiosurgery. Ann Thorac Surg
2015;100:1163-5; discussion 1165-6.

24. Cheng SL, Chu CM. Electromagnetic navigation bronchoscopy under intravenous sedation-tips and tricks. J Thorac Dis 2018;10:S769-74.

25. Petersen J, Wille MMW, Rakêt LL, et al. Effect of inspiration on airway dimensions measured in maximal inspiration CT images of subjects without airflow limitation. Eur Radiol 2014;24:2319-25.

26. Sun J, Xie F, Zheng X, et al. Learning curve of electromagnetic navigation bronchoscopy for diagnosing peripheral pulmonary nodules in a single institution. Transl Cancer Res 2017;6:541-51.

27. Chan JWY, Lau RWH, Ng CSH. Electromagnetic navigation bronchoscopy fiducial marker margin identification plus triple dye for complete lung nodule resection. JTCVS Techniques 2020;3:329-33.

28. Kupelian PA, Forbes A, Willoughby TR, et al. Implantation and stability of metallic fiducials within pulmonary lesions. Int J Radiat Oncol Biol Phys 2007;69:777-85.

(English Language Editor: B. Draper)

Cite this article as: Chen J, Pan X, Gu C, Zheng X, Yuan H, Yang J, Sun J. The feasibility of navigation bronchoscopyguided pulmonary microcoil localization of small pulmonary nodules prior to thoracoscopic surgery. Transl Lung Cancer Res 2020;9(6):2380-2390. doi: 10.21037/tlcr-20-1206 